\title{
Are psychiatric comorbidities and associated cognitive functions related to treatment response to methylphenidate in boys with attention-deficit/ hyperactivity disorder?
}

\author{
This article was published in the following Dove Press journal: \\ Neuropsychiatric Disease and Treatment \\ II April 2017 \\ Number of times this article has been viewed
}

\author{
Mandy H Chan' \\ Patrick WL Leung² \\ Ting-pong $\mathrm{Ho}^{3}$ \\ Se-fong Hung ${ }^{4}$ \\ Chi-chiu Lee ${ }^{5}$ \\ Chun-pan Tang 5 \\ Ka-chai Cheung ${ }^{2}$ \\ Fung-yee Ching ${ }^{2}$ \\ Fefe HK Chan ${ }^{6}$ \\ Lu-hua Chen ${ }^{2}$ \\ Merce Garcia-Barcelo ${ }^{7}$ \\ Pak-chung Sham ${ }^{3}$ \\ 'Department of Clinical Psychology, \\ Kowloon Hospital, Hospital Authority, \\ ${ }^{2}$ Department of Psychology, The \\ Chinese University of Hong Kong, \\ ${ }^{3}$ Department of Psychiatry, University \\ of Hong Kong, ${ }^{4}$ Department of \\ Psychiatry, The Chinese University \\ of Hong Kong, ${ }^{5}$ Department of \\ Psychiatry, Kwai Chung Hospital, \\ Hospital Authority, ${ }^{6}$ Department of \\ Clinical Psychology, Alice Ho Mui \\ Ling Nethersole Hospital, Hospital \\ Authority, ${ }^{7}$ Department of Surgery, \\ University of Hong Kong, Hong Kong, \\ People's Republic of China
}

Correspondence: Patrick WL Leung Department of Psychology, The Chinese University of Hong Kong, 3/F, Sino

Building, Shatin, Hong Kong, People's

Republic of China

Tel +8523943 6502

Fax +852 26035019

Email pleung@cuhk.edu.hk
Background: Methylphenidate (MPH) has been found to be an effective medication for attention-deficit/hyperactivity disorder (ADHD). However, there are neither consistent nor sufficient findings on whether psychiatric comorbidities and associated cognitive functions of ADHD are related to treatment response to MPH in ADHD children.

Objectives: This study investigated whether psychiatric comorbidities, IQ, and neurocognitive deficits are related to treatment response to MPH in ADHD children. In some ways, it is preferable to have a drug that the effectiveness of which to a disorder is not affected by its associated cognitive functions and psychiatric comorbidities. On the other hand, it is likely that the baseline symptom severity of ADHD is associated with the effectiveness of MPH treatment on the symptoms post treatment.

Methods: A total of 149 Chinese boys (aged 6-12 years) with ADHD, combined type, and normal IQ participated in this study. Assessment of ADHD symptom severity was conducted pre and post MPH treatment, while assessment of psychiatric comorbidities, IQ, and neurocognitive deficits was performed in a non-medicated condition. Treatment response was defined as the ADHD symptom severity post MPH treatment.

Results: Results indicated that MPH treatment was effective, significantly improving the ADHD condition. Yet, comorbid disorders, IQ, and neurocognitive deficits were not related to MPH treatment response on ADHD symptoms. These findings indicated that the effectiveness of MPH was not affected by psychiatric comorbidities and associated cognitive functions of ADHD. Instead, as expected, it was the baseline symptom severity that was mainly related to the treatment response, ie, the milder the baseline condition, the better the treatment response.

Conclusion: The current findings positively endorse the widespread clinical use of MPH for treating ADHD. It improves the behavioral symptoms of ADHD regardless of varying psychiatric comorbidities, IQ, and neurocognitive deficits.

Keywords: ADHD, psychiatric comorbidities, cognitive functions, treatment response, methylphenidate

\section{Introduction}

Attention-deficit/hyperactivity disorder (ADHD) is characterized by inattention (IA), hyperactivity, and impulsivity, incurring considerable learning and psychosocial impairments. It is among the most prevalent neuropsychiatric disorders affecting $5 \%-10 \%$ of children worldwide. ${ }^{1}$ Despite that existing stimulant treatment (primarily by methylphenidate, MPH) falls short of a cure to eradicate ADHD, it remains the 
most efficacious treatment for short-term symptomatic relief of ADHD with effect sizes ranging from 0.78 to $0.96 .^{2,3}$ Yet, it is also known that there is individual variation in response to $\mathrm{MPH}$ among ADHD children with a minority, up to $20 \%-30 \%$, reporting milder or little improvement. ${ }^{4}$ Unfortunately, there are neither consistent nor sufficient findings on conditions differentiating responsiveness to $\mathrm{MPH}$ in ADHD children. In this study, a comprehensive range of such potential conditions was examined.

\section{Psychiatric comorbidities of ADHD Anxiety}

Initial studies found that response to MPH would be poorer among ADHD children with anxiety. 5,6 There was suspicion that ADHD with comorbid anxiety might be different from that without. ${ }^{7}$ The inattentive behavior of the former might actually be secondary to anxiety. However, recent studies reported that comorbid anxiety made no difference to the effectiveness of MPH with ADHD. ${ }^{8,9}$

\section{Depressive disorders}

Studies found inconsistent results regarding comorbid depressive disorders on treatment response to MPH with ADHD. Yet once again, recent studies seemed to support that ADHD with or without comorbid depressive symptoms equally benefited from MPH. ${ }^{10,11}$

\section{Oppositional defiant disorder (ODD) and conduct disorder (CD)}

Quite a number of studies found that ADHD children with or without comorbid ODD/CD responded equally well to MPH. ${ }^{8,12}$ One study found that ADHD children with comorbid aggressive behaviors responded better to MPH. ${ }^{6}$ However, the study itself explained that the better response might be due to inflated rating consequential to halo effects brought upon by improvement in disruptive and oppositional behaviors.

\section{Autism spectrum disorder (ASD)}

The few studies available found that ADHD children with or without ASD responded equally well to MPH. ${ }^{13,14}$

\section{Cognitive functions IQ}

Available studies showed that ADHD children with mental retardation did not respond well to $\mathrm{MPH} .{ }^{15,16}$ However, MPH was equally effective for ADHD children whose IQs were within the normal range. ${ }^{17}$ It was reasoned that ADHD with mental retardation might represent a qualitatively different type of the disorder than that with normal intelligence. ${ }^{18}$

\section{Specific learning difficulties}

The impact of comorbid specific learning difficulties on treatment response to MPH in ADHD children has been scarcely studied. Available literature indicated that they were not related to treatment response to $\mathrm{MPH} .{ }^{19}$

\section{Neurocognitive deficits}

There are a number of neurocognitive deficits identified to be associated with ADHD, namely response disinhibition, interference control dysfunction, delay aversion, poor working memory, time estimation errors, and deficient sustained attention. However, their relationship to treatment response to MPH is rarely studied. The few studies that were available showed inconsistent findings. Response disinhibition was related to poorer treatment response to MPH in ADHD children, while interference control dysfunction was not related to any differences in MPH treatment response. ${ }^{20,21}$ Given such scanty literature, it is difficult to conclude whether ADHD-associated neurocognitive deficits are related to treatment response to $\mathrm{MPH}$ in ADHD children.

\section{Objectives}

Given the existing inconsistent and limited findings, there is no strong evidence to conclude either way as to whether psychiatric comorbidities and associated cognitive functions are related to treatment response to MPH in ADHD children. The current study aimed at reexamining this question, including a wide range of psychiatric comorbidities and cognitive functions. In some ways, it is preferable to have a drug that the effectiveness of which to a disorder is not affected by its associated cognitive functions and frequent psychiatric comorbidities. The lack of such relationships should be welcomed by clinicians who find the drug (MPH) equally beneficial to ADHD children with diverse psychiatric comorbidities and cognitive functions. On the other hand, it is likely that the baseline symptom severity of the disorder (ADHD) is associated with the effectiveness of $\mathrm{MPH}$ in alleviating the symptoms post treatment.

\section{Methods}

\section{Participants}

The sample was recruited from boys attending a child psychiatric clinic in Hong Kong between January 2013 and September 2015 who were clinically diagnosed with ADHD (combined type) by psychiatrists, based on the Diagnostic and Statistical Manual of Mental Disorders, Fourth Edition, Text Revision (DSM-IV-TR). ${ }^{22}$ The current study recruited only boys because of the high gender ratio of boys to girls with ADHD presented in child psychiatric clinics. ${ }^{23}$ The number 
of girls might still be too small even within a reasonably sized sample of over 100 for data analysis in a scientifically acceptable way. Additional inclusion criteria were Chinese ethnicity, aged 6-12 years, and studying in local mainstream primary schools. Exclusion criteria were IQ below 80, bipolar disorder, psychosis, autism, severe obsessive-compulsive disorder, Tourette's syndrome or chronic serious tics, birth injury, head trauma, or major causative genetic, neurological, metabolic, or infectious illnesses.

\section{Measures}

Wechsler Intelligence Scale for Children - fourth edition (Hong Kong) (WISC-IV [HK])

It is a test of intelligence for children with local norms for Hong Kong. Four subtests, the scores of which had been found to be highly correlated with the full-scale IQ, were administered, ie, similarities, digit span, matrix reasoning, and coding. ${ }^{24}$

\section{National Institute of Mental Health (NIMH) Diagnostic Interview Schedule for Children - fourth edition (parent-informant) (P-DISC-IV)}

It is a structured diagnostic interview with parents as informants and is administered by trained personnel. It examines more than 30 common child psychiatric disorders. P-DISC-IV was well validated as a diagnostic tool with both clinical and community samples. ${ }^{25}$ It generates categorical diagnoses as well as dimensional symptom scores (criterion counts).

\section{Chinese version of the Autism Spectrum Quotient - I0 items (child version) (Chinese AQ- I0-Child)}

The Chinese AQ-10-Child is a parent-report questionnaire for assessing autistic features in children. It is an abbreviated version of the 50-item Autism Spectrum Quotient (child version). It had been found to show high sensitivity and specificity in screening ASD. ${ }^{26}$

\section{Hong Kong Test of Specific Learning Difficulties in Reading and Writing for Primary School Students - second edition (HKT-P[II])}

It is a test of specific learning difficulties for Cantonesespeaking children with local norms for Hong Kong. The HKT$\mathrm{P}(\mathrm{II})$ had reported satisfactory psychometric properties. ${ }^{27}$

\section{The stop-signal task}

It is a computerized reaction time task of response inhibition. Participants are required to withhold a response by not pressing a key if a tone is sounded in a particular trial. The measure reflecting participants' capability of response inhibition is called "stop-signal reaction time (SSRT)". It estimates how long it takes for a participant to inhibit a response. Stop-signal task had demonstrated satisfactory validity in differentiating between ADHD children and normal controls. ${ }^{28}$

\section{The Stroop color-word task (computerized version)} It is a task of interference control. An interference score, Golden interference score,${ }^{29}$ measures the participants' ability to suppress a prepotent or habitual response.

Within-subject variability in reaction time in the Stroop color-word task is regarded as a measure assessing sustained attention. It is operationalized as within-subject standard deviation of reaction time (SD-RT) and coefficient of variation (CV). A larger SD-RT and CV indicate a greater variability in responses, reflecting a difficulty in sustaining attention to maintain a constant speed to respond. The SD-RT was found to show high sensitivity and specificity in discriminating ADHD individuals from normal controls. ${ }^{30}$

\section{The Maudsley Index of Childhood Delay Aversion (MIDA)}

It is an index indicating delay aversion in children with ADHD. Participants are required to make a choice between a smaller immediate reward, ie, a shorter-sooner (SS) response, earning 1 point if they only choose to wait for 2 seconds, or a larger delayed reward, ie, a larger-later (LL) response, earning 2 points if they instead choose to wait for 30 seconds. There are two experimental conditions: one with a post reward delay and one without. MIDA was found to be significantly related to ADHD. ${ }^{31}$

\section{Digit Span subtest of WISC-IV (HK)}

It is used to examine working memory of the participants. Children with ADHD had been found to perform significantly poorer in the Digit Span subtest when compared to normal controls. ${ }^{32}$

\section{Time estimation task}

This task assesses participants' ability to accurately estimate the length of different time intervals randomly presented to them, namely $6,12,36$, and 60 seconds. An absolute discrepancy (AD) score is computed, which is the absolute difference between participants' estimated time and actual time presented to them. ${ }^{33}$

\section{Strengths and Weaknesses of ADHD Symptoms and Normal Behaviors Rating Scale (SWAN) (parent- informant)}

It is a parent-report questionnaire that has been revalidated in Hong Kong and provides rating of the 18 key ADHD 
features on children. ${ }^{34}$ A lower score represents a severer ADHD condition. In this study, the SWAN provides assessment of the baseline symptom severity of ADHD pre MPH treatment as well as the treatment response to MPH (indexed by the post MPH treatment SWAN score). This definition of treatment response is commonly used in previous studies, including the landmark Multimodal Treatment of ADHD (MTA) study in the USA. ${ }^{14,35}$

\section{Procedures}

The psychiatrists at their regular consultation sessions determined the clinic attendants who matched the study's inclusion and exclusion criteria. For those who met the former and not the latter, invitation was made to their parents to participate in the study. Written informed consent was provided by the parents of the participants for this study. This study obtained ethics approval from relevant institutional boards, namely Joint Chinese University of Hong Kong - New Territories East Cluster Clinical Research Ethics Committee and Kowloon West Cluster Research Ethics Committee.

All clinic attendants referred to our child psychiatric clinic would be asked to fill in a number of questionnaires, including SWAN, when they came to their first consultation session. For the ADHD boys recruited to participate in our study, those SWAN records were taken as assessing the baseline pre-MPH treatment ADHD symptom severity. The ADHD boys were then arranged to undergo testing on IQ, neurocognitive deficits, and specific learning difficulties. The participants' parents served as informants to the DISC-IV. The assessment with one ADHD participant and one of his parents as an informant required separately 2-3 hours each. Despite efforts to schedule the assessment sessions as soon as possible, they might still take a month or two to arrange, since local children had usually a very busy schedule, given full-day schooling, heavy homework, and a very competitive examination system. However, since MPH was considered the first-line treatment of ADHD in Hong Kong, it would be prescribed even at the first consultation once the case psychiatrists had clinically established the ADHD diagnosis. Therefore, despite that a sizable portion of our participants were new referrals, they were likely to be on MPH treatment when assessment sessions were finally scheduled. To have baseline assessment of their cognitive functions unaffected by medication, all ADHD participants were required to be medication free for at least 48 hours prior to the testing.

All participants in this study received MPH treatment for their ADHD condition. Based upon the case psychiatrists' clinical expertise, some participants $(53 \%)$ in our study were prescribed multiple-dose, immediate-release $\mathrm{MPH}$, namely Ritalin, while some other participants were prescribed a single-dose, extended-release MPH, namely Concerta or Ritalin long-acting (LA) (16.8\% and 10.7\%, respectively). The remaining participants (19.5\%) were prescribed both Concerta and Ritalin or Ritalin and Ritalin LA. The dosages from Concerta and Ritalin LA were converted into dosage units of Ritalin for data compilation according to an international standard. ${ }^{36}$ In sum, the dosage of all participants ranged from 10 to $60 \mathrm{mg}$ per day (Ritalin; mean =23.43; $\mathrm{SD}=9.48$ ). Specifically, $87.9 \%$ of the participants $(n=131)$ were prescribed $10-30 \mathrm{mg}$ of MPH per day, while only $12.1 \%$ of the participants $(n=18)$ were prescribed over $30 \mathrm{mg}$ per day.

SWAN was readministered as the outcome measure of treatment response after at least 12 weeks of continual MPH treatment. This 12-week period was considered to be of sufficient duration to allow proper titration for all participants, based upon the clinical judgment of the case psychiatrists at regular follow-ups. No other form of treatment was concurrently received by the participants.

\section{Statistical analyses}

Statistical analyses were conducted using SPSS (version 22.0; IBM Corp., Armonk, NY, USA). Baseline ADHD symptom severity was predicted to be related to treatment response to MPH so that it would be treated as a covariate in analysis (analyses of covariance [ANCOVA] or partial correlation) examining the relationship between psychiatric comorbidities/ cognitive functions and treatment response. The latter was operationalized in this study as the post MPH treatment SWAN score. The abovementioned analysis would be followed by regression analysis, entering those significantly related independent variables to build a prediction model.

\section{Results}

A total of 264 clinically diagnosed ADHD boys of Chinese ethnicity were recruited into the study. Among them, the clinical diagnosis of 77 children failed to be confirmed by DISC-IV as ADHD, combined type. Many of these 77 children were instead diagnosed by DISC-IV as either the IA or hyperactivity-impulsivity (HI) type of ADHD. This lack of perfect agreement between clinical diagnosis and DISC-IV diagnosis was not entirely unexpected, given the known moderate inter-rater reliability of psychiatric diagnosis. The loss of sample size was compensated by an increased confidence on the diagnostic identity of our ADHD participants. A total of 27 children were further excluded because of IQs below 80 . Of the 160 children who were 
eligible for this study, three children were eventually not treated by MPH. Eight children defaulted the MPH treatment at various time points during the 12 -week titration period. The final sample of this study was thus composed of 149 boys. Figure 1 depicts graphically the multiple steps in arriving at our final sample.

There was a significant difference between pre and post MPH treatment SWAN scores, indicating a positive treatment effect of MPH (mean $=44.7, \mathrm{SD}=11.8$ vs mean $=69.8$, $\mathrm{SD}=17.3, t[148]=17.57$, Cohen's $d=1.50$ ). As predicted, there was a significant correlation between pre and post MPH treatment SWAN scores; milder baseline ADHD symptom severity was associated with better treatment response on ADHD symptoms post MPH treatment $(r=0.34 ; P<0.001)$. In view of space limitation, descriptive statistics of other variables (mean and SD) are not reported in this study, but can be made available upon request from the corresponding author.

The psychiatric comorbidities tested included social phobia, separation anxiety disorder, agoraphobia, selective mutism, obsessive-compulsive disorder, specific phobia, major depressive disorder, dysthymic disorder, ODD, and CD. Panic disorder, generalized anxiety disorder, and post traumatic stress disorder were assessed but not tested, since no ADHD children had obtained such diagnoses from DISC-IV. Since many individual diagnoses had small numbers, they were collapsed into broader diagnostic groupings, namely any anxiety disorder (including all anxiety disorders assessed in DISC-IV), any depressive disorder (including major depressive disorder and dysthymic disorder), any disruptive

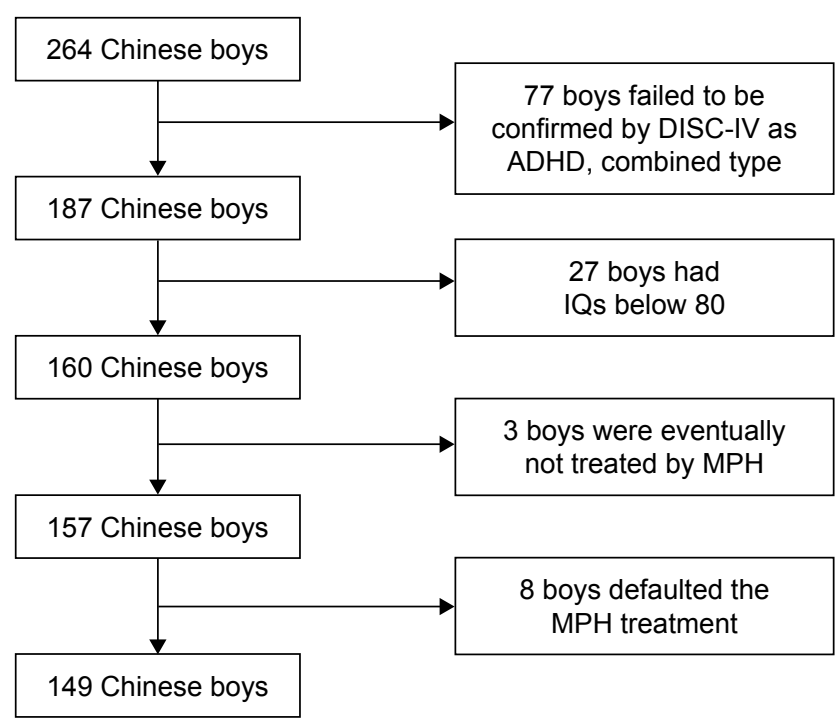

Figure I Flowchart of recruitment of participants.

Abbreviations: ADHD, attention-deficit/hyperactivity disorder; DISC-IV, Diagnostic Interview Schedule for Children - fourth edition; MPH, methylphenidate. disorder (including ODD and CD), and any psychiatric disorder (including all DISC-IV diagnoses tested). These broader groupings gave more balanced ratios between those ADHD children with some of these comorbidities and those without. There was uniformly no significant difference in treatment response between groups with or without various comorbidities (Table 1).

Dimensional analysis by partial correlation (controlling baseline ADHD symptom severity) between DISC-IV criterion counts of various comorbidities and post MPH treatment SWAN score was also performed and found the same insignificant results. The correlation coefficients were uniformly very small $(<0.15$, most even at $<0.10$; Table 2$)$. The comorbidities tested included not only all DISC-IV diagnoses mentioned earlier but also specific learning difficulties and ASD symptoms, whose measures provide dimensional scores. Broad diagnostic groupings were again created by summing the criterion count of each disorder after proration. However, the same lack of significant correlation with very small coefficients was found $(<0.10$; Table 2$)$.

There was also no significant partial correlation between ADHD symptoms (SWAN) post MPH treatment and associated cognitive functions, after partialing out the baseline ADHD symptom severity (Table $3 \mathrm{~A}$ and $\mathrm{B}$ ). The correlation coefficients were again very small $(<0.15$, most even at $<0.10$ ). The cognitive functions tested included single or multiple indices of IQ, response disinhibition, interference control dysfunction, deficient sustained attention, poor working memory, delay aversion, and time estimation errors.

Multiple regression was not run to build a prediction model, since no independent variable was found to be correlated with treatment response to MPH in ADHD symptoms.

\section{Discussion}

All psychiatric comorbidities of ADHD tested in this study, including individual disorders and broader diagnostic groupings, were not related to treatment response to MPH in ADHD symptoms. The lack of such relationship is consistent with the literature regarding comorbid specific learning difficulties and ASD. In this study, we must note that those children with a clinical diagnosis of ASD have already been excluded from inclusion. However, a minority of the ADHD participants (10\%) still scores above the clinical cutoff of AQ. Hence, our current sample can still provide some range of ASD symptoms to test how their presence affects the ADHD outcome of MPH treatment.

On the other hand, some previous studies do suggest that comorbid anxiety, depression, and ODD/CD may affect the 
Table I ADHD symptoms (SWAN) post MPH treatment between groups with and without psychiatric comorbidities (N=I49)

\begin{tabular}{|c|c|c|c|c|c|c|}
\hline \multirow{3}{*}{$\begin{array}{l}\text { Psychiatric } \\
\text { comorbidities }\end{array}$} & \multirow{2}{*}{\multicolumn{2}{|c|}{$\begin{array}{l}\text { With DISC diagnosis } \\
\text { Post MPH treatment } \\
\text { SWAN }\end{array}$}} & \multirow{2}{*}{\multicolumn{2}{|c|}{$\begin{array}{l}\text { Without DISC diagnosis } \\
\text { Post MPH treatment } \\
\text { SWAN }\end{array}$}} & \multirow{2}{*}{\multicolumn{2}{|c|}{ Statistics }} \\
\hline & & & & & & \\
\hline & Mean & SD & Mean & SD & Mann-Whitney Ua & $P$-value \\
\hline Social phobia & $67.35(n=13)$ & 21.11 & $70.11(n=136)$ & 17.06 & 855.50 & 0.85 \\
\hline SAD & $59.50(n=5)$ & 25.04 & $70.09(n=143)$ & 17.05 & 265.00 & 0.33 \\
\hline Agoraphobia & $72.25(n=2)$ & 33.59 & $69.83(n=147)$ & 17.28 & 136.00 & 0.86 \\
\hline Selective mutism & $86.50(n=3)$ & 14.00 & $69.52(n=146)$ & 17.32 & 98.50 & 0.10 \\
\hline OCD & $81.38(n=4)$ & 11.24 & $69.58(n=145)$ & 17.45 & 168.50 & 0.15 \\
\hline Major depression & $73.00(n=2)$ & 4.95 & $69.82(n=147)$ & 17.50 & 127.50 & 0.76 \\
\hline Dysthymic disorder & $65.71(n=7)$ & 22.56 & $70.07(n=142)$ & 17.17 & 447.50 & 0.66 \\
\hline$C D$ & $64.77(n=11)$ & 20.97 & $70.27(n=138)$ & 17.10 & 622.50 & 0.32 \\
\hline \multirow[t]{2}{*}{ Any depressive disorder } & $67.33(n=9)$ & 19.88 & $70.03(n=140)$ & 17.28 & 600.00 & 0.81 \\
\hline & & & & & $F^{\mathrm{b}}$ & df (P-value) \\
\hline Specific phobia & $69.08(n=50)$ & 18.80 & $70.26(n=99)$ & 16.7| & 0.29 & I (0.59) \\
\hline ODD & 71.7I (n=83) & 17.83 & $67.53(n=66)$ & 16.66 & 1.68 & $\mathrm{I}(0.20)$ \\
\hline Any anxiety disorder & $69.30(n=60)$ & 18.77 & $70.25(n=89)$ & 16.49 & 0.06 & $\mathrm{I}(0.8 \mathrm{I})$ \\
\hline Any disruptive disorder & $71.57(n=85)$ & 17.55 & $67.61(n=64)$ & $|6.5|$ & 1.67 & I $(0.20)$ \\
\hline Any psychiatric disorder & $71.35(n=107)$ & 17.90 & $66.10(n=42)$ & 15.58 & 3.24 & I (0.07) \\
\hline
\end{tabular}

Notes: No ADHD participant was diagnosed with panic disorder, GAD, and PTSD by DISC-IV so that there was no test of their relationship to treatment response to $\mathrm{MPH}$. ${ }^{\mathrm{M}}$ Mann-Whitney $U$ test was conducted to compare groups with and without psychiatric comorbidities because the data were not normally distributed, except for any psychiatric disorder, any anxiety disorder, any disruptive disorder, specific phobia, and ODD when ${ }^{\mathrm{D}} \mathrm{ANCOVA}$ was conducted because of their data meeting the normality requirement, controlling the baseline SWAN score as a covariate. Post MPH treatment SWAN, SWAN score post MPH treatment; any psychiatric disorder is any anxiety, depressive, or disruptive disorder. Some statistical computations were based $<149$ participants due to some missing data.

Abbreviations: ADHD, attention-deficit/hyperactivity disorder; ANCOVA, analyses of covariance; CD, conduct disorder; DISC-IV, Diagnostic Interview Schedule for Children - fourth edition; GAD, generalized anxiety disorder; MPH, methylphenidate; OCD, obsessive-compulsive disorder; ODD, oppositional defiant disorder; PTSD, post traumatic stress disorder; SAD, separation anxiety disorder; SD, standard deviation; SWAN, Strengths and Weaknesses of ADHD Symptoms and Normal Behaviors Rating Scale.

Table 2 Partial correlation of ADHD symptoms post MPH treatment with psychiatric comorbidities, controlling baseline ADHD symptoms $(\mathrm{N}=149)$

\begin{tabular}{lllllllllllllllllllll}
\hline $\begin{array}{l}\text { Psychiatric } \\
\text { comorbidities }\end{array}$ & $\mathbf{1}$ & $\mathbf{2}$ & $\mathbf{3}$ & $\mathbf{4}$ & $\mathbf{5}$ & $\mathbf{6}$ & $\mathbf{7}$ & $\mathbf{8}$ & $\mathbf{9}$ & $\mathbf{1 0}$ & $\mathbf{1 1}$ & $\mathbf{1 2}$ & $\mathbf{1 3}$ & $\mathbf{1 4}$ & $\mathbf{1 5}$ & 16 & 17 & 18 & 19 \\
\hline Post-tx SWAN & 0.05 & 0.03 & -0.03 & 0.05 & 0.09 & 0.09 & -0.03 & 0.07 & 0.12 & -0.01 & 0.12 & 0.08 & -0.09 & 0.06 & -0.06 & 0.05 & 0.03 & $-0.01^{\mathrm{a}}$ & 0.14 \\
\hline
\end{tabular}

Notes: Nonparametric partial correlation computed due to the non-normality of the independent variables except the one marked by the footnote letter "a" in superscript, when its data fulfilled the normality requirement; Post-tx SWAN, SWAN score post MPH treatment; variables I-I7 being criterion count of DISC-IV diagnoses: I, any psychiatric disorder; 2 , any anxiety disorder; 3, any depressive disorder; 4, any disruptive disorder; 5, social phobia; 6, separation anxiety disorder; 7, specific phobia; 8, panic disorder; 9, agoraphobia; 10, generalized anxiety disorder; II, selective mutism; 12, obsessive-compulsive disorder; I3, post traumatic stress disorder; I4, major depressive disorder; 15, dysthymic disorders; 16, ODD; I7, CD; 18, literacy score from the subtests of HKT-P(II), ie, Chinese word reading and Chinese word dictation; 19, autism quotient. Some statistical computations were based $<149$ participants due to some missing data.

Abbreviations: ADHD, attention-deficit/hyperactivity disorder; CD, conduct disorder; DISC-IV, Diagnostic Interview Schedule for Children - fourth edition; HKT-P(II), Hong Kong Test of Specific Learning Difficulties in Reading and Writing for Primary School Students - second edition; MPH, methylphenidate; ODD, oppositional defiant disorder; SWAN, Strengths and Weaknesses of ADHD Symptoms and Normal Behaviors Rating Scale.

Table 3A Partial correlation of ADHD symptoms post MPH treatment with IQ, response disinhibition (stop-signal task), interference control dysfunction (Stroop task), and deficient sustained attention (Stroop task), controlling baseline ADHD symptoms ( $\mathrm{N}=149$ )

\begin{tabular}{llllllllll}
\hline $\begin{array}{l}\text { IQ, response disinhibition, } \\
\text { interference control dysfunction, } \\
\text { deficient sustained attention }\end{array}$ & $\mathbf{I}$ & $\mathbf{2}$ & $\mathbf{3}$ & $\mathbf{4}$ & $\mathbf{5}$ & $\mathbf{6}$ & $\mathbf{7}$ & $\mathbf{8}$ & $\mathbf{9}$ \\
\hline Post-tx SWAN & & & & & & & & & \\
\hline
\end{tabular}

Notes: Nonparametric partial correlation computed due to non-normality of the data of the independent variables; Post-tx SWAN, SWAN score post MPH treatment; I, estimated IQ from four subtests of WISC-IV (HK), ie, similarities, digit span, coding, and matrix reasoning; 2, SSRT (stop-signal task); 3, Golden interference score (indicating interference control dysfunction, Stroop task); variables 4-9 from Stroop task indicating deficient sustained attention: 4, word reading: SD-RT; 5, color naming: SD-RT; 6, incongruent color naming of color words: SD-RT; 7, CV in word reading; 8, CV in color naming; 9, CV in incongruent color naming of color words. Some statistical computations were based $<149$ participants due to some missing data.

Abbreviations: ADHD, attention-deficit/hyperactivity disorder; CV, coefficient of variation; MPH, methylphenidate; SD-RT, standard deviation of reaction time; SSRT, stop-signal reaction time; SWAN, Strengths and Weaknesses of ADHD Symptoms and Normal Behaviors Rating Scale; WISC-IV (HK), Wechsler Intelligence Scale for Children - fourth edition (Hong Kong). 
Table 3B Partial correlation of ADHD symptoms post MPH treatment with delay aversion (MIDA task), time estimation errors (time estimation task) and poor working memory (Digit Span of WISC-IV [HK]), controlling baseline ADHD symptoms (N=I49)

\begin{tabular}{lllllllllll}
\hline $\begin{array}{l}\text { Delay aversion, time estimation } \\
\text { errors, and poor working memory }\end{array}$ & $\mathbf{1}$ & $\mathbf{2}$ & $\mathbf{3}$ & $\mathbf{4}$ & $\mathbf{5}$ & $\mathbf{6}$ & $\mathbf{7}$ & $\mathbf{8}$ & $\mathbf{9}$ & $\mathbf{1 0}$ \\
\hline Post-tx SWAN & 0.03 & -0.08 & 0.05 & -0.11 & -0.08 & -0.08 & -0.01 & 0.02 & -0.03 & 0.01 \\
\hline
\end{tabular}

Notes: Nonparametric partial correlation computed due to non-normality of the data of the independent variables; Post-tx SWAN, SWAN score post MPH treatment; variables I-3 from the MIDA task indicating delay aversion: I, percentage of SS response under the condition of without post reward delay; 2, percentage of SS response under the condition of with post reward delay; 3, MIDA; variables 4-7 from time estimation task indicating time estimation errors: 4, AD of 6-second time interval; 5, AD of I2-second time interval; 6, AD of 36-second time interval; 7, AD of 60-second time interval; variables 8-10 from Digit Span of WISC-IV (HK) indicating poor working memory: 8, scaled score of Digit Span (WISC-IV [HK]); 9, scaled score of Digit Span Forward; I0, scaled score of Digit Span Backward. Some statistical computations were based $<149$ participants due to some missing data.

Abbreviations: AD, absolute discrepancy; ADHD, attention-deficit/hyperactivity disorder; MIDA, Maudsley Index of Childhood Delay Aversion; MPH, methylphenidate; SS, shorter sooner; SWAN, Strengths and Weaknesses of ADHD Symptoms and Normal Behaviors Rating Scale; WISC-IV (HK), Wechsler Intelligence Scale for Children - fourth edition (Hong Kong).

degree of benefits from MPH treatment. However, it must be noted that as a whole, existing evidence supporting these findings is neither strong nor consistent. More recent studies with comorbid anxiety and depression seem to suggest that ADHD children with or without these comorbidities equally benefit from MPH. Although one study does find improved response to MPH by ADHD children with aggressive behavior, it reasons that the improvement is mainly due to inflated rating influenced by halo effects. ${ }^{6}$ Expectedly, existing studies differ from each other in terms of research designs, measures, definitions of disorders or treatment response, samples, etc. It cannot be easily concluded whether these differences may explain some of the inconsistent findings. More studies are probably required in the future to see the dominant trend of findings emerging from studies of diverse methodologies and populations.

Consistent with the existing literature that IQ within the normal range is not related to treatment response to $\mathrm{MPH}$ in ADHD symptoms,${ }^{17}$ our study with participants having IQ of 80 or above shows a similar finding.

Response disinhibition, interference control dysfunction, poor working memory, delay aversion, time estimation errors, and deficient sustained attention are identified neurocognitive deficits of ADHD. All of their measures administered in a non-medicated condition in the current study are not related to treatment response to MPH in ADHD symptoms. Given the very limited existing studies noted earlier, it is hard to decide whether our current findings are to be expected or not. Once again, more replication studies are called for.

Besides behavioral symptoms, MPH has also been found to improve neurocognitive functioning, such as sustained attention and working memory, ${ }^{37}$ and normalize the malfunctioned brain regions associated with ADHD, eg, increasing the activities of frontal and striato-thalamic regions. ${ }^{38}$ On the other hand, ADHD behavioral symptoms and neurocognitive deficits may not be causally related to each other directly. ${ }^{39}$ Changes in neurocognitive functioning do not necessarily bring corresponding changes in behavioral symptoms of ADHD or vice versa. In other words, baseline non-medicated neurocognitive functioning may be related to improvement in its own domain post MPH treatment, but not necessarily to behavioral improvement in ADHD symptoms, as measured by our outcome measure, SWAN. In future studies, a fairer examination of the relationship between baseline neurocognitive deficits and MPH treatment response is to have measures of neurocognitive outcomes post MPH treatment. However, for informing clinical practice, changes in behavioral symptoms remain the primary concern for clinicians. This explains why this study chooses to concentrate on behavioral symptoms of ADHD rather than its neurocognitive functioning.

\section{Limitations and future direction}

First, this study does not pretend to be a clinical trial; it has not adopted the standard methodology of a randomized controlled trial (RCT). There are neither standardized dosages nor duration of treatment for the ADHD participants. Instead, it is a naturalistic clinic study with boys who attended a child psychiatric clinic for routine treatment of their ADHD condition. The case psychiatrists use their clinical expertise to determine the optimal MPH treatment for each boy. The only condition that this study imposes on the ADHD treatment is to allow the psychiatrists at least 12 weeks to identify the optimal dosage before post MPH treatment SWAN is administered to determine the treatment response. We take this clinic ADHD treatment to examine whether psychiatric comorbidities and non-medicated cognitive functions of ADHD can predict treatment response to MPH in ADHD symptoms. The behavioral improvement that we see from the clinical practice of MPH medication is encouraging, but we will not claim this finding a formal evaluation of the efficacy of MPH without conducting a well-controlled RCT. 
Our reported improvement on ADHD condition post MPH treatment should not be interpreted as such. Nonetheless, a large effect size of improvement should not be considered as unexpected, given converging evidence of repeated metaanalyses from many previous well-controlled clinical trials. ${ }^{2,3}$ It is less likely that the treatment response is an artifact resulting from regression-to-mean, maturation, or a learning effect from repeated assessment, etc.

The current study included participants who are boys aged 6-12 years with IQ of 80 or above and ADHD, combined type. The results of this study may not be generalizable to ADHD girls or to ADHD boys with other types of ADHD (ie, IA or HI), with ages before 6 years or beyond 12 years, or with IQ below 80 . The same concern applies to ADHD children with a range of psychiatric comorbidities excluded from this study (our exclusion criteria are stated earlier). In addition, our coverage of conditions potentially influencing MPH treatment response is not exhaustive. For example, negative self-concept was recently found to be a predictor of poorer treatment responsiveness to MPH. ${ }^{40}$ Future research should expand to cover the aforementioned untested conditions.

This study used a parent-report measure, SWAN, for assessing treatment response. Having multiple informants may be desirable to obtain a more comprehensive picture of the ADHD behaviors under assessment. We should also consider assessing functioning and behaviors other than those primary symptoms of ADHD, such as social or academic functioning, as treatment responses in future study.

Last but not least, there is so far limited knowledge about the underlying pharmacological mechanism of MPH. This makes our current study explorative rather than theoretically driven. We may have to wait for more knowledge about the therapeutic mechanism of MPH before we can have a more informed choice of conditions to be tested as related to treatment response to MPH. Nonetheless, the current findings imply that the drug action of MPH seems to be quite exclusively related to ADHD. Its effectiveness does not seem to be dependent on or in interaction with those frequent psychiatric comorbidities and associated cognitive functions of ADHD. Thus, it is not surprising to find the baseline ADHD symptom severity as the only condition correlated with the treatment response to $\mathrm{MPH}$ in ADHD children.

\section{Conclusion and clinical implication}

This study has its strength and contribution. It covers a more comprehensive list of potential conditions to be related to response to MPH treatment in ADHD children than those of many previous studies. Some of our tested conditions, such as neurocognitive deficits, have been rarely studied and some have previously produced inconsistent findings. Our study is also reasonably sized with 149 well-defined children with ADHD, combined type. The results have also been very uniform with the group comparison and correlation consistently insignificant with small coefficients across all clinical and cognitive conditions tested. This is so despite a large number of statistical tests having been conducted and this may have caused concern of chance findings if significant results did emerge.

The lack of relationship between treatment response to MPH and psychiatric comorbidities/associated cognitive functions in fact positively endorses the widespread clinical use of MPH for treating ADHD. It improves the behavioral symptoms of ADHD regardless of varying psychiatric comorbidities and associated cognitive functions. Given the clinical reality of high frequencies of psychiatric comorbidities and neurocognitive deficits with ADHD, clinicians are pleased to learn that they do not affect the clinical effectiveness of MPH. For example, at one time, studies seem to suggest that MPH treatment is not effective for ADHD children with comorbid anxiety. However, this study joins the more recent findings indicating that comorbid anxiety makes no difference to the effectiveness of MPH treatment to ADHD. Given this growing literature, clinicians can confidently prescribe MPH to ADHD children with comorbid anxiety so that they can also reap the therapeutic benefits of MPH.

\section{Acknowledgment}

This study is funded by a General Research Fund (GRF) grant to the corresponding author, Patrick WL Leung, from the Research Grants Council (RGC), Hong Kong (RGC 449511).

\section{Disclosure}

The authors report no conflicts of interests in this work.

\section{References}

1. Swanson JM, Sergeant JA, Taylor EA, Sonuga-Barke EJS, Jensen PS, Castellanos FX. Attention Deficit Hyperactivity Disorder. In: Pfaff DW, Volkow ND, editors. Neuroscience in the 21st Century. New York, NY: Springer Science + Business Media; 2016:2-18.

2. Faraone SV, Glatt SJ. A comparison of the efficacy of medications for adult attention-deficit/hyperactivity disorder using meta-analysis of effect sizes. J Clin Psychiatry. 2010;71(6):754-763.

3. Leucht S, Hierl S, Kissling W, Dold M, Davis JM. Putting the efficacy of psychiatric and general medicine medication into perspective: review of meta-analyses. Br J Psychiatry. 2012;200(2):97-106.

4. Schachter HM, Pham B, King J, Langford S, Moher D. How efficacious and safe is short-acting methylphenidate for the treatment of attention deficit disorder in children and adolescents? A meta-analysis. CMAJ. 2001; 165(11):1475-1488. 
5. Moshe K, Karni A, Tirosh E. Anxiety and methylphenidate in attention deficit hyperactivity disorder: a double-blind placebo-drug trial. Atten Defic Hyperact Disord. 2012;4(3):153-158.

6. Ter-Stepanian M, Grizenko N, Zappitelli M, Joober R. Clinical response to methylphenidate in children diagnosed with attention-deficit hyperactivity disorder and comorbid psychiatric disorders. Can J Psychiatry. 2010;55(5):305-312.

7. Goez H, Back-Bennet O, Zelnik N. Differential stimulant response on attention in children with comorbid anxiety and oppositional defiant disorder. J Child Neurol. 2007;22(5):538-542.

8. Jensen PS, Hinshaw SP, Kraemer HC, et al. ADHD comorbidity findings from the MTA study: comparing comorbid subgroups. J Am Acad Child Adolesc Psychiatry. 2001;40(2):147-158.

9. Abikoff H, McGough J, Vitiello B, et al; RUPP ADHD/Anxiety Study Group. Sequential pharmacotherapy for children with comorbid attention-deficit/hyperactivity and anxiety disorders. J Am Acad Child Adolesc Psychiatry. 2005;44(5):418-427.

10. DuPaul GJ, Barkley RA, McMurray MB. Response of children with ADHD to methylphenidate: interaction with internalizing symptoms. J Am Acad Child Adolesc Psychiatry. 1994;33(6):894-903.

11. Gadow KD, Nolan EE, Sverd J, Sprafkin J, Schwartz J. Anxiety and depression symptoms and response to methylphenidate in children with attention-deficit hyperactivity disorder and tic disorder. $J$ Clin Psychopharmacol. 2002;22(3):267-274.

12. Pliszka S. The effects of anxiety on cognition, behavior, and stimulant response in ADHD. J Am Acad Child Adolesc Psychiatry. 1989;28(6): 882-887.

13. Santosh PJ, Baird G, Pityaratstian N, Tavare E, Gringras P. Impact of comorbid autism spectrum disorders on stimulant response in children with attention deficit hyperactivity disorder: a retrospective and prospective effectiveness study. Child Care Health Dev. 2006;32(5): 575-583.

14. Handen BL, Johnson CR, Lubetsky M. Efficacy of methylphenidate among children with autism and symptoms of attention-deficit hyperactivity disorder. J Autism Dev Disord. 2000;30(3):245-255.

15. Aman MG, Buican B, Arnold LE. Methylphenidate treatment in children with borderline IQ and mental retardation: analysis of three aggregated studies. J Child Adolesc Psychopharmacol. 2003;13(1):29-40.

16. Pearson DA, Lane DM, Santos CW, et al. Effects of methylphenidate treatment in children with mental retardation and ADHD: individual variation in medication response. J Am Acad Child Adolesc Psychiatry. 2004;43(6):686-698

17. Efron D, Jarman F, Barker M. Methylphenidate versus dextroamphetamine in children with attention deficit hyperactivity disorder: a double-blind, crossover trial. Pediatrics. 1997;100(6):E6.

18. Rutter M, Macdonald H, Le Couteur A, Harrington R, Bolton P, Bailey A. Genetic factors in child psychiatric disorders - II. Empirical findings. J Child Psychol Psychiatry. 1990;31(1):39-83.

19. Tannock R, Ickowicz A, Schachar R. Differential effects of methylphenidate on working memory in ADHD children with and without comorbid anxiety. J Am Acad Child Adolesc Psychiatry. 1995;34(7):886-896.

20. Scheres A, Oosterlaan J, Sergeant JA. Speed of inhibition predicts teacherrated methylphenidate response in boys with $\mathrm{AD} / \mathrm{HD}$. Int J Disabil Dev Educ. 2006;53(1):93-109.

21. van der Oord S, Geurts HM, Prins PJ, Emmelkamp PM, Oosterlaan J. Prepotent response inhibition predicts treatment outcome in attention deficit/hyperactivity disorder. Child Neuropsychol. 2012;18(1): $50-61$.

22. American Psychiatric Association. Diagnostic and Statistical Manual of Mental Disorders. 4th ed., text rev. Washington, DC: American Psychiatric Association; 2000.

23. Graetz BW, Sawyer MG, Hazell PL, Arney F, Baghurst P. Validity of DSM-IV ADHD subtypes in a nationally representative sample of Australian children and adolescents. J Am Acad Child Adolesc Psychiatry. 2001;40(12):1410-1407.
24. Wechsler D. Wechsler Intelligence Scale for Children. 4th ed. (short form). Hong Kong, China: King-May Psychological Assessment; 2010.

25. Shaffer D, Fisher P, Lucas CP, Dulcan MK, Schwab-Stone ME. NIMH Diagnostic Interview Schedule for Children Version IV (NIMH DISC-IV): description, differences from previous versions, and reliability of some common diagnoses. J Am Acad Child Adolesc Psychiatry. 2000; 39(1):28-38.

26. Allison C, Auyeung B, Baron-Cohen S. Toward brief "Red Flags" for autism screening: the short autism spectrum quotient and the short quantitative checklist in 1,000 cases and 3,000 controls. J Am Acad Child Adolesc Psychiatry. 2012;51(2):202-212.

27. Ho CSH, Chan DW, Tsang S, Lee S. The Hong Kong Test of Specific Learning Difficulties in Reading and Writing (HKT-SpLD) Manual. Hong Kong: Hong Kong Specific Learning Difficulties Research Team; 2007.

28. Oosterlaan J, Logan GD, Sergeant JA. Response inhibition in AD/HD, $\mathrm{CD}$, comorbid $\mathrm{AD} / \mathrm{HDCD}$, anxious, and control children: a metaanalysis of studies with the stop task. J Child Psychol Psychiatry. 1998; 39(3):411-425.

29. Golden C. Stroop Color and Word Test: A Manual for Clinical and Experimental Uses. Chicago, IL: Stoelting; 1978.

30. Uebel H, Albrecht B, Asherson P, et al. Performance variability, impulsivity errors and the impact of incentives as gender-independent endophenotypes for ADHD. J Child Psychol Psychiatry. 2010;51(2): 210-218.

31. Paloyelis Y, Asherson P, Kuntsi J. Are ADHD symptoms associated with delay aversion or choice impulsivity? A general population study. $J$ Am Acad Child Adolesc Psychiatry. 2009;48(8):837-846.

32. Mayes SD, Calhoun SL. Learning, attention, writing, and processing speed in typical children and children with ADHD, autism, anxiety, depression, and oppositional-defiant disorder. Child Neuropsychol.2007; 13(6):469-493.

33. Hui J. Sense of Time and Delay Aversion in Attention Deficit/ Hyperactivity Disorder (ADHD) [Unpublished master's thesis]. Hong Kong: Department of Psychology, Chinese University of Hong Kong; 2007.

34. Chan GFC, Lai KYC, Luk ESL, Hung SF, Leung PWL. Clinical utility of the Chinese strengths and weaknesses of ADHD-symptoms and normal-behaviors questionnaire (SWAN) when compared with DISC-IV. Neuropsychiatr Dis Treat. 2014;10:1533-1542.

35. Owens EB, Hinshaw SP, Kraemer HC, et al. Which treatment for whom with ADHD? Moderators of treatment response in the MTA. J Consult Clin Psychol. 2003;71(3):540-552.

36. U.S. Food and Drug Administration. Full prescribing information for Concerta; 2010. Available from: http://www.accessdata.fda. gov/drugsatfda_docs/label/2010/021121s026s027lbl.pdf. Accessed January 27, 2017.

37. Agay N, Yechiam E, Carmel Z, Levkovitz Y. Methylphenidate enhances cognitive performance in adults with poor baseline capacities regardless of attention-deficit/hyperactivity disorder diagnosis. J Clin Psychopharmacol. 2014;34(2):261-265.

38. Pauls AM, O’Daly OG, Rubia K, Riedel WJ, Williams SC, Mehta MA. Methylphenidate effects on prefrontal functioning during attentionalcapture and response inhibition. Biol Psychiatry. 2012;72(2): $142-149$.

39. Coghill DR, Hayward D, Rhodes SM, Grimmer C, Matthews K. A longitudinal examination of neuropsychological and clinical functioning in boys with attention deficit hyperactivity disorder (ADHD): Improvements in executive functioning do not explain clinical improvement. Psychol Med. 2014;44(5):1087-1099.

40. Say GN, Karabekiroglu K, Yuce M. Factors related to methylphenidate response in children with attention deficit/hyperactivity disorder: a retrospective study. Düşünen Adam. 2015;28(4):319-327. 


\section{Publish your work in this journal}

Neuropsychiatric Disease and Treatment is an international, peerreviewed journal of clinical therapeutics and pharmacology focusing on concise rapid reporting of clinical or pre-clinical studies on a range of neuropsychiatric and neurological disorders. This journal is indexed on PubMed Central, the 'PsycINFO' database and CAS, and is the official journal of The International Neuropsychiatric Association (INA). The manuscript management system is completely online and includes a very quick and fair peer-review system, which is all easy to use. Visit http://www.dovepress.com/testimonials.php to read real quotes from published authors.

\footnotetext{
Submit your manuscript here: http://www.dovepress.com/neuropsychiatric-disease-and-treatment-journal
} 